Aus dem anatomisch-histologischen Laboratorium der Universität St. Petersburg.

\title{
Zur Frage über den Bau des Plexus chorioideus.
}

Von

W. Hworostuchin.

Hierzu Tafel X.

In zahlreichen histologischen Arbeiten kommen die Autoren neuerer Zeit einstimmig zum Ergebnis der sekretorischon Tütigkeit des Epithels, welches die Plexus chorioidei bedeckt; diese Befunde werden auch durch Versuche eines ver'starkten Zutlusses der cerebrospinalen Flüssigkeit bestätigt. Die vielfachen Untersuchungen aus der letzten Zeit beweisen, dass die Forschungen auf diesem Gebiet energisch fortgesetzt werden, doch sind viele Seiten der Frage entweder noch gar nicht oder nur unvollständig untersucht worden.

Auf Rat meines hochverehrten Lehrers, Herrn Prof. Dr. A.S. Dogiel, habe ich versucht, so viel als möglich diese Lücken auszutüllen. Ich spreche ihm hier meinen Dank ans!

\section{Kurze Übersicht der neueren Literatur.}

Ich verweise zunächst auf die Arbeit von Galeotti (1897). Beim Studium der vergleichenden Anatomie des Diencephalon verschiedener Wirbeltiere vermerkte er die sekretorische Tätigkeit des Epithels der Plexus chorioidei. Den Sekretionsprozess selber hat er recht ausführlich beschrieben. Im Kern sollen sich kleine Granula bilden, die später ins Protoplasma übertreten und zur Peripherie der Zelle rücken. Beim Abrücken derselben vom Kern nehmen sie allmählich an Grösse zu und werden schliesslich in die Gehirnventrikel ausgeschieden. Ferner beobachtete Galeotti die Umwandlung fuchsinophiler Granula in Pigmentkörner. Find lay (1899) hält gleich Gal e otti die Plexus für sekretorische Organe. Er sah im Protoplasma der Epitheizellen äberall zahlreiche homogene Sekretgranula, im apikalen Abschnitt einiger Zellen jedoch Vakuolen, welche infolge einer Zerstörung der Zellmembran in den Liquor cerebrospinalis übergingen.

Studnicka (1900) kunnte beim Studium des Ependyms bei verschiedenen rorwiegend niederen Wirbeltieren die Ausstossung der Sekrettropfen nicht nur aus dem Epithel der Plexus chorioidei, sondern auch aus den Ependymzellen verschiedener Abschnitte der Gehirnventrikel (Wandungen der Paraphyse, Fossa rhomboidea u. a.) beobachten. Diese Beobachtungen 
veranlassten ihn, die Vermutung auszusprechen, dass das ganze Ependym sich an der Sekretion beteilige. Hierbei nimmt er jedoch an, dass in Berücksichtigung der histologischen Struktureigentümlichkeiten der einzelnen Ependymabschnitte auch ein Unterschied in den Sekretprodukten erwartet werden müsse.

Fast zu denselben Ergebnissen wie Gale otti gelangte auch Francini (1907). Unter anderem beschrieb er in den Epithelzellen des Plexus chorioideus Tropfen mit intensiv gefärbtem Saume.

Cavazzani (1893), Paul Clais se et Charles Levi (1897) und L o e per (1904) studierten den Plexus chorioideus in pathologischen Fällen und gelangten hinsichtlich seiner sekretorischen Tätigkeit zu positiven Ergebnissen.

Cappelleti (1901), Petit et Girard (1901) und II eek (1907) konstatierten bei Einwirkung von Pilokarpin. Muskarin und anderer Substanzen eine Znnahme der Cerebrospinalflüssigkeit, wobei $P$ etit et $G i$ rard sowie Y e e k an dem nach den Versuchen fixierten Material ausserdem charakteristische Veränderungen im Epithel der Plexus gesehen haben. Petit et Girard schreiben hierüber folgendermassen: „la hauteur des éléments épitheliaux s'accroit, la differenciation en denx zones s'exagére, la zone distale prend un développement exagéré et la production des globules hialins devient plus active qu' it l'état normal; en an mot, ces éléménts hypersécrètent." Fast dasselbe vermerkt auch $\mathrm{Me}$ e $\mathrm{k}$ : , a differentiation inte two zones, a basal granular, and an outer clear... The granulations, however, are always heavier and more compact toward the base of the cell. Clear spaces begin to appear toward the lop, and rarely dues the stainable cytoplasm extend to the upper cell wall. Masses of larger grannles are common in the upper part of the cell where the lines forming the reticulations cross."

Ohne mich ausführlich bei einer Reihe anderer Arbeiten von Imamura, Loeper, Schläpfer, Joschimura u. a. aufzuhaiten, will ich nur einige interessante Angaben aus denselben hier wiedergeben. I $\mathrm{m}$ a $\mathrm{m} \mathrm{u}$ ra (1902) beschreibt glänzende, fettartige Köıner, welche eine Reaktion mit Osmiumsäure ergeben. Lo e p e r (1904) beobachtete Glylogenkörner sowie kleine und grössere fettartige Gebilde, die häufig das Aussehen einer Morula haben; sie werden durch Äther, Xylol gelöst, während Osmiumsäure sie schwach färbt. Schläpfer (1905) nahm Sekrettropfen mit lipoider Hülle wahr. Joschimura (1909) schliesslich fand in den Epithelzellen des Plexus Lecithin, Fett, Fibrin und Glykogen.

\section{Material und Technik.}

Ich studierte die Plexus chorioidei hauptsächlich an fixiertem Material, das ich verschiedenen Säugetieren (Katze, Maus, Kaninchen, Hase, Pferd, Affe u. a.) unter normalen Bedingungen entnahm. Ausserdem untersuchte ich auch lebendes Gewebe in cerebrospinaler Flüssigkeit oder in physiologischer Kochsalzlösung. Zur Fixierung versuchte ich viele der in der mikroskopischen Technik gebränchlichsten Gemische, wobei die Mehrzahl derselben sich untanglich erwies. Einige derselben enthielten keine Osmiumsäure, infolgedessen zablreiche fettähnliche Einschlüsse in dem Epithel 
unsichtbar blieben (Gemische von Car noy-Gil s on: Lenhos ék, konzentrierte Sublimatlösung in physiologischer Kochsalzlösung $u$. a.); andere Gemische enthielten Osmiumsäure, doch auch eine grosse Menge Essigsäure, welche, soviel ich beurteilen kann, Veränderungen in diesem zarten Organ verursachte (die Mitochondrien löste $u$. a.). Die besten Resultate ergab die Fixierung der Präparate nach $A 1 \mathrm{tmann}$ und abgeänderte Verfahren dieser $\left(1^{0} \%\right.$ Osmiumsäure und $2^{1}: 2^{0} ; 0$ Kaliumbichromatlïsung zu gleichen Teilen). Gewöhnlich fixierte ich die Präparate 24 Stunden lang, wusch sie darauf in Wasser ans und härtete sie in Alkohol von steigender Konzentration, schloss sie in Paraffin ein und fertigte aus ihnen Schnitte von $3-4$ "Dicke an. Zur Fürbung benutzte ich hauptsüichlich saures Fuchsin und Hämatoxylin nach $\mathrm{Heide} \mathrm{nhain}$.

\section{Bau des Epithels der Plexus chorioidei.}

Meine Untersuchungen bestätigen teilweise die Beobachtungen der angeführten Forscher, teilweise ergeben sie neue Befunde, die ich nachstehend beschreiben werde.

Die Form der Epithelzellen, welche die Oberttäche des Plexus chorioideus des vierten Ventrikels und der Seitenventrikel bekleiden, ist ăusserst mannigfaltig. Gewöhnlich weisen die Zellen kubische Form auf, oder ihr Längsdurchmesser ist beträchtlich grösser als der Querdurchmesser oder umgekehrt der Querdurchmesser ist grösser als der Langsdurchmesser (Fig. I), Bereits diese Grössenschwankungen deL Zellen geben Veranlassung zur Annahme, dass hierbei der Funktionszustand eine gewisse Rolle spielt, obgleich beim Studium eines dermassen zarten Objektes, wie es die Plexus chorioidei sind, die Möglichkeit einer Formveründerung der Zelle durch rein mechanische Ursachen, wie z. B. durch Zerrung der Membran während der Präparation u. dgl. ins Auge gefasst werden muss.

Bereits bei füchtiger Durchsicht der Präparate ist es jedoch nicht schwer, in den einzelnen Zellen eine wechselnde Menge von Granula wahrzunehmen (Fig. I), wahrend bei einer genauen Beobachtung ein gewisser Unterschied in dem Bau der Granula erkannt werden kann.

So fand ich häufig im Epithel, welches den Plexus chorioideus der Seitenventrikel und des vierten Gehirnventrikels der Katze auskleidet (nach einer Fixierung desselben in modifiziertem Altmannschen Gemisch mit nachfolgender Hamatoxylinfarbung nach Heidenbain) kubische Zellen, deren Protoplasma eine betrăchtliche Menge körniger Fäden, welche ihrer Form und ihrer 
Lage nach an Mitochondrien erinnern, sowie eine geringe Anzahl einzelner Crranula enthielten. Diese körnigen Fäden haben gewöhnlich Komma-, Bogen- oder Stäbchenform, doch werden auch wellenförmige Fäden verschiedener Länge angetroffen. Diese Gebilde sind in der ganzen Zelle verstreut, wenngleich sie in grösserer Zahl neben dem Kern im distalen Teil der Zelle sich vorfinden (Fig. II und Fig. III, Zelle 2).

In einigen Zellen derselben Form ist umgekehrt eine grössere Henge isolierter Körner und eine unbedeutende Anzahl von Fäden rorhanden. Die Körnchen selber sind jn diesen Fälen verschieden gross (Fig. III, Zelle 1 und 3; Fig. VI, Zelle 2). Derartige Bilder habe ich auch bisweilen auf Praparaten gesehen, die nach dem Originalverfaluren von Altmann behandelt worden waren.

Fernerhin werden auf Präparaten, die nach denselben Verfabren bearbeitet worden sind. Zellen angetroffen, in denen nur kleine, mehr oder weniger gleichmässig gefärbte Granula, häufig in dermassen grosser Anzahl, dass die ganze Zelle von ihnen angefüllt zu sein scheint, sich vorfinden. Die Granula sind in diesen Fallen hauptsichlich im distalen Zellabschnitt, sowie zu beiden Seiten des Kerns angeordnet; bisweilen jedoch werden einige Granula auch unterhalb des Kerns angetroften (Fig. VII, Zelle 1. Fig. IV, Fig. V, Zelle 4).

Ausserdem sind auch Bilder wie folgt sichtbar: die ganze Zelle von mehr oder weniger linglicher form ist dicht angefült von Granulis der verschiedensten Form und der verschiedensten Färbungsintensitat; dieselben sind unregelmåssig in der Zelle verstreut, wobei die grössten und am stärksten gefürbten Granulain dem distalen Zellteil sich vorfinden (Fig. V, Zelle 7; Fig. VI. Zelle 1 und 3).

In anderen hohen Zellen werden neben kleinen homogenen Granulis bisweilen bauptsïchlich in der Nähe des Zellgipfels besondere grosse ,Granula mit hellem Zentrum" oder „Tropfen mit stark gefarbtem Saum", wie sie von einigen Forschern bezeichnet werden, angetroffen (Fig. V, Zelle 3 und 6; Fig. VII, Zelle 2). Sie erinnern einigermassen an die von M. Heidenhain in der Beckendrüse von Triton helveticus, von $\mathrm{Nicolas}$ in den Trănendrüsen sowie von anderen Forschern in Drüsen mit flüssiger Sekretion beschriebenen "Halbmondkörperchen".

Am häufigsten finden sich jedoch in den hohen Zellen ausser verschiedenen Granulaarten noch Vakuolen. Sie sind gewöhnlich 
neben den "Granulis mit hellem Zentrum“ gelegen und infolgedessen bisweilen schwer von ihnen zu unterscheiden (Fig. V, Zelle 1 und 2; Fig. VII, Zelle 3 und 4).

Wie aus der vorliegenden Darstellung hervorgeht, ist zwischen der Zellform und der Struktur derselben eine gewisse Gesetzmässigkeit vorhanden. Diese Gesetzmässigkeit kann natürlich nur als allgemeine Regel, als Bilder, die am bäufigsten anzutreffen sind, angesehen werden; in seltenen Fallen fand ich jedoch auch Ausnahmen von dieser Regel, die jedoch eine Erklärung zuliessen.

Die vermerkte Gesetzmässigkeit, das Vorhandensein von Mitochondrien und Halbmondkörperchen geben meiner Meinung nach einen Hinweis auf den Sekretionsmechanismus des Plexusepithels. Soviel ich beurteilen kann, verläutt der Sekretionsprozess folgendermassen: die einzelnen Chondriomitenköıner nehmen an Umfang zu. als năhmen sie Nahrmaterial aus dem Protoplasma auf; derartige Körner treten in stets zunehmender Zahl auf, während die Zahl der Chondriomiten abnimmt. Weiterhin nehmen die Körner (Granula) an Grösse zu, in ihnen geht ein komplizierter Prozess vor sich, der sich äusserlich durch eine intensivere Färbung der Granula mit saurem Fuchsin und Hämatoxylin nach $\mathrm{Heide} \mathrm{nhain}$ dokumentiert. Alsdann folgt gleichsam eine Lösung und Umwandlung derselben in Sekrettropfen. Von der Richtigkeit der hier dargelegten Annahme werde ich überzeugt durch die in letzter Zeit angestellten Untersuchungen an verschiedenen typischen Drüsen. So fanden Regaud et Mawas (1909) Mitochondrien in der Parotis und der Submaxillaris und vermerkten ibre Beteiligung an der Sekretionstätigkeit, dasselbe beschrieb auch Regaud (1909) in der Niere, Policard (1909) und Fiessinger (1909) in der Leber u. a.

Unwillkürlich taucht nun die Frage auf, was denn die Epithelzellen des Plexus chorioideus vorbereiten?

Auf meinen nach Altma in s Verfahren behandelten Präparaten sind fast immer in den Zellen Körner zweierlei Art sichtbar. Die einen derselben farben sich mit saurem Fuchsin und haben die Form und die Grösse typischer Seluretgranula; andere färben sich mit Osmiumsaure schwarz (bei Kaninchen und Hasen), oder dunkelgrau (beim Pferd) oder hellgrau mit einem gelblichen Farbenton (bei Katzen u. a.); ihre Grösse und Form ist äusserst verschieden. In einigen Zellen sind sie klein und einzeln ver- 
streut (Fig. VIII; Fig. I, Zelle d); in anderen liegen derartige Körner in Gruppen, vorwiegend zu vier, zusammen. Ausser kleinen Lïrnern und einzelnen kleinen Anhäufungen derselben sind in den Zellen auch grössere Granula sichtbar (Fig. I). Am häufigsten besonders bei Katzen, Pferden, Hasen und Kaninchen werden grosse, bald homogene, bald körnige, kugelförmige Gebilde, oder wie sie von einigen Autoren bezeichnet werden "Gebilde von Morulaform" angetroffen (Fig. V, Zelle 1 und 3; Fig. I, Zelle $c$; Fig. VII).

In welchem Wechselverbältnis beide Arten von Granula stehen, habe ich nicht feststellen können; ich will nur vermerken, dass in Zellen, in denen viele fuchsinophile Granula vorhanden sind, die mit Osmiumsäure gefärbten kugelförmigen Gebilde nur in geringer Zahl sichtbar sind, nicht selten nur ein grosses Gebilde im basalen Teil der Zelle.

Auf Grund meiner Beobachtungen kann ich mit Bestimmtheit aussagen, dass $\mathrm{Me} \mathrm{ek}$ mit seiner Behauptung, als würden die grossen kugelförmigen Gebilde unter normalen Bedingungen nur bei Kaninchen angetroffen, nicht im Recht ist; unrichtig sind meiner Meinung nach auch seine Schlüsse über zweierlei Arten von Sekretion. Es ist schwer, anzunehmen, dass bei allen von mir untersuchten Tieren (Katzen, Pferden, Hasen u. a.) die Epithelzellen des Plexus chorioideus sich unter anormalen Bedingungen befunden haben. Diese Gebilde haben ausserdem auch viele andere Forscher beschrieben, wobei sie am hauufigsten für Tropfen einer fettähnlichen Substanz gehalten wurden (Lo eper, Engel [1909], Imamura); Galeotti und einige andere erklarten sie für Pigment, Josch i m u r a für Lecithin. Auf Grund einer Reihe von Reaktionen, in Berücksichtigung der Lösung der Substanz der kugelförmigen Gebilde in Alkobol und Äther, ihres Verhaltens zu Osmiumsaure und zu der speziellen Reaktion von Ciacciol), halte ich es für das wahrscheinlichste, dass in den Bestand dieser Gebilde Lecithin eingeht.

Galeotti, Engel und einige andere Autoren beschreiben noch besondere grosse basophile Gebilde. Ich sah sie nur ein-

1) Das Nachweisverfahren von Lecithin nach $\mathrm{Ciaccio}$ gründet sich auf die Tatsache, dass das Lecithin nach einer Behandlung mit alkalischen Bicbromaten in den gewöhnlichen Fettlösungsmitteln unlöslich wird. Diese Reaktion ergab jedoch bei mir nicht immer günstige Resultate. 
bis zweimal in Präparaten, die nach Hermann und Galeotti fixiert und mit saurem Fuchsin und Lichtgrün gefürbt worden waren. Zunächst hielt ich sie für grosse basophile Gebilde, bei einer genaueren Untersuchung und nach Fixierung von Teilen desselben Stückes in verschiedenen Flüssigkeiten habe ich mich davon überzeugt, dass hier dieselben fettïhnlichen (Lecithin) Gebilde vorliegen, welche ich soeben beschrieben habe.

Auf ungefärbten, in Hermannscher Flüssigkeit fixierten Präparaten erscheinen sie matt, auf solchen, die mit Lichtgrün gefärbt waren, weisen sie einen leicht grünlichen Ton, wie das gesamte Protoplasma, auf. Es liegt kein Grund vor, anzunehmen, dass die basophilen Granula bei der Behandlung zerstört werden, da das fixierte Material dasselbe Strukturbild rom Epithel des Plexus chorioideus ergibt, wie frisches Gewebe (Fïg. XII).

Am Schlusse der Beschreibung der sekretorischen Erscheinungen im Epithel muss ich noch die Frage über die Beteiligung des Kernes an diesem Prozesse berühren. Eine unmittelbare Beteiligung des Kerns an der Bildung der Sekretgranula, wie sie einige Autoren beschreiben (Galeotti u. a.), habe ich nicht gesehen. Ich kann nur angeben, dass ich Kernveränderungen in verschiedenen Phasen der sekretorischen Tätigkeit der Zellen gesehen habe, jedoch keinerlei Gesetzmüssigkeit festzustellen vermochte. Ich lasse daher diese Frage offen. Das einzige, was ich über die Kerne in den Epithelzellen des Plexus chorioideus aussagen kann, ist, dass ich vielfach beim Hasen, seltener bei Affen, noch seltener bei Katzen, Pferden und anderen Tieren, zweikernige Zellen wahrgenommen habe (Fig. IX; Fig. I, Zelle b). In einigen Fällen berührten sich die Kerne (Fig. X). Da ich in den Kernen keine Mitosen gesehen habe, einige Bilder jedoch auf eine direkte Teilung hinwiesen, so ist die Annahme zulässig, dass sich die Kerne hier amitotisch teilen.

Håufiger als auf Schnitten habe ich zweikernige Zellen auf Flächenpräparaten nach Behandlung derselben mit Methylenblau gesehen. In ein bis zwei Fallen habe ich (beim Hasen) dreikernige Zellen gesehen (Fig. IX).

Soviel mir bekannt ist, ist eine Teilung der Epithelzellen und speziell zwei- und dreikerniger Zellen von niemand früher im Epithel des Plexus chorioideus vermerkt worden. 


\section{Nerven der Plexus chorioidei.}

Nachdem ich mich von der sekretolischen Tratigkeit des den Plexus chorioideus bedeckenden Epithels überzeugt hatte, interessierte mich die Frage über die Ausbreitung der Śerven in diesem Organ. Literaturangaben über diese Nerven sind nur wenige vorhanden; sie geben keine positive Antwort auf die gestellte Frage. In der alteren Arbeit von Benedikt (1874) sind nur einige Hinweise darauf vorhanden, dass in dem Plexus chorioideus des vierten Ventrikels Äste vom Nervus vagus teils mit den Blutgefïssen, teils jedoch anscheinend zum Epithel des Plexus verlaufen.

Einige neue Befunde ergaben die Arbeiten ron Findlay (18.99) und Bochenek (1899). Find lay hat entgegen B o chenek nur vaso-motorische Nervenfasern gesehen, wilhrend letzterer beim Frosch in der Paraphyse ein grosses Geflecht auf den Blutgefässen beschreibt, welches Ästchen zum Plexus chorioideus der Seitenventrikel abgibt. Die das Geflecht bildenden Nerven stammen, wie Bochenek nachweisen konnte, aus den die Carotis cerebralis umflechtenden Plexus sympathicus.

Aus dem Mitgeteilten geht hervor, dass noch niemand ausfuihrlich die Nervenverteilung in den P'lexus chorioidei selber verfolgt hat, infolgedessen ich mich bemüht habe, diese Frage vermittels einer Fürbung der Nerven mit Nethylenblau klarzustellen. Ich fürbte hierbei folgendermassen: am Gehirn eines frisch getöteten Tieres öfnete ich vorsichtig die Gehirnventrikel. Ich entfernte die übertlüssigen (iehirnteile, wobei ich nur diejenigen Gehirnabschnitte unberülurt liess, welche dem Plexus chorioideus anliegen; darauf feuchtete ich die Obertlache des letzteren mit einer geringen Menge einer schwachen Methylenblaulösung $(1 / 8 \%-1 / 16 \%)$ an, worauf das Präparat in einem Thermostaten auf eine für verschiedene Tiere verschiedene Zeit aufgestellt wurde; von Zeit zu Zeit kontrollierte ich den Verlauf der Färbung unter dem Mikroskop. Ich fixierte die Präparate nach der Fărbung in molybdänsaurem Ammonium.

Mein Hauptaugenmerk richtete ich auf das Studium der Nerven des Plexus chorioideus des vierten Ventrikels und der Seitenventrikel. In dem Teil des Plexus chorioideus des dritten Ventrikels, welcher dem Foramen Monroi gegenüber liegt, habe ich ein grosses breitmaschiges Geflecht aus sehr dicken mark- 
haltigen und marklosen Nervenfasern gesehen. Im Plexus chorioideus des Seitenventrikels habe ich an verschiedenen Stellen ein breitmaschiges Geflecht aus markhaltigen und marklosen Fasern angetroffen; nirgends waren dieselben jedoch so dick, wie in dem angegebenen Geflecht des dritten Ventrikels. Bisweilen habe ich wahrnebmen können, dass von einem Ast des Geflechts sich feinere Zweige absondern, die ihrerseits in noch feinere zerfielen. Letztere verflechten sich miteinander, wobei das gebildete Geflecht unmittelbar unter dem Epithel des Plexus liegt (Fig. XIII).

In einigen Fallen konnte ich feststellen, dass von einigen Ïstchen des subepithelialen Getlechts feine Fadchen abgingen, die auf der Obertläche der Epithelzellen endigten. Ausser diesem $G$ eflecht werden natürlich stets von ihnen deutlich unterschiedliche Geflechte auf den zahlreichen Blutgefässen angetroffen.

In dem Plexus chorioideus des vierten Ventrikels sah ich den Eintritt dicker Nervenfasern mit der Arteria cerebelli inferior posterior; auch in ihm habe ich wie in dem Plexus der Seitenventrikel hautig zarte subepitheliale Getlechte sowie Getlechte auf den Blutgefissen beobachtet.

$$
\text { Ependym. }
$$

Bei der Fixierung ganzer Gehirne kleiner Tiere batte ich Gelegenheit, auch den Bau des Ependyms des Seitenventrikels kennen zu lernen. Auf derartigen Präparaten konnte ich in den Ependymzellen deutlich sowohl fuchsinophile Granula als auch Vakuolen wahrnehmen. Diese Bilder bestatigen die von Studnička (1900) ausgesprochene Annahme von einer sekretorischen Tätigkeit überhaupt des Ependyms der Gehirnhöhlen.

\section{Ergebnisse.}

Das Studium des feineren Baues des den Plexus chorioideus des Seitenventrikels und des vierten Gehirnventrikels bedeckenden Epithels ergibt positive Resultate hinsichtlich der Beteiligung derselben an der Bildung des Liquor cerebrospinalis.

In den ruhenden Drüsenzellen des Plexus chorioideus sowie in den frühen Sekretionsstadien sind Mitochondrien vorhanden, hinsichtlich derer Gründe vorliegen, sie für identisch mit den vegetativen Faden $A l t m$ an $n$ s zu halten. In den spateren Sekretionsstadien sind in den Epithelzellen Gebilde sichtbar, die an die Halbmondkörperchen von $\mathrm{M}$. $\mathrm{He}$ ide $\mathrm{nh}$ a in erinnern. 
Zahlreiche Nervenfasern bilden im Plexus chorioideus gröbere und feinere Geflechte sowohl auf den Blutgefässen als auch unterbalb des Plexusepithels, wobei vom subepithelialen Geflecht feinste Fadchen abgehen, welche auf der Oberfliache der Epithelzellen endigen.

Die Epithelzellen des Plexus chorioideus enthalten gewöhnlich einen Kern, doch werden auch zwei- und dreikernige Zellen angetroffen, wobei die Teilung des lierns augenscheinlich durch Amitose erfolgt.

Hinsichtlich der von den Drüsenzellen ausgearbeiteten Produkte gelang es mir nur festzustellen, dass unter ihnen Lecithin vorhanden ist. 


\section{Literaturverzeichnis.}

Benedikt): Über die Innervation des Plexus chorioideus inferior. S c h midt s Jahrbücher, 1874.

B ochenek: Über die Nervendigangen in den Plexus chorioidei des Frosches. Bull. inter. de l'Acad. de Cracovie, Nr. 7, 1899.

Cappelleti'): L'ecoulement du liquide cerebrospinal par la fistule cephalorach. en conditions norm. et sous l'influence de quelques medicaments. Arch. Ital. de Biol., Vol. 36, 1901.

Cavazzani'): Sur la circulation du liquide cerebro-spinal. Arch. Ital. de Biol., Vol. 18, 1893.

$\mathrm{Ciaccio:} \mathrm{Über} \mathrm{das} \mathrm{Vorkommen} \mathrm{von} \mathrm{Lecithin} \mathrm{in} \mathrm{den} \mathrm{cellularen} \mathrm{Ent-}$ zündungsprodukten und über besondere lipoidbildende Zellen (Lecithinzellen). Zentralbl. Allg. Path., 1909, Bd. 20, Nr. 9.

Champy: A propos de mitochondries des cellules glandulaires et des cellules renales. Comp. rend. de la Société Biol., 1909.

Enge 1: Über die Sekretionserscheinung in den Zellen der Plexus chorioidei des Menschen. Arch. f. Zellforsch., H. I, 1908.

Fiessinger: Contributions a l'étude des degenerescen. de la cellule hepatique. Comp. rend. d. l. Soc. Biol., 1909.

Findlay: The choroid plexus of the lateral ventricles of the brain. Brain Nr. 86, Vol. 22, 1899.

Francin $\left.i^{1}\right)$ : Sulla struttura e la funzione dei plessi corioidei. Lo sperimentale., Bd. LXI, 1907.

Ga le otti: Studio morfologico e citologico della volta del'diencephalo in acluni vertebrati. Riviste di Patolng. nerv. e ment, 1897.

Derselbe: Û̉ber die Granulationen in den Zellen. Int. Monatsschr. f. Anat. u. Phys., Bd. XII, 1895.

$\mathrm{H}$ a e ckel: Beiträge zur normalen und pathologischen Anatomie der Plexus chorioid. Vir ch ow s Arch., Bd. 16, 1859.

He ide $n$ ha in: Plasma und Zellen. 1907.

I ma mura: Beiträge zur Histologie des Plexus chorioideus des Menschen. Arb. a. d. Neurol. Inst. a. d. Wien. Univ., H. VIII, 1902.

Joschimura: Das histochemische Verhalten des menschlichen Plexus chorioideus. Arb. a. d. Neurol. Inst. a. d. Wien. Univ., Bd. 18, H. I, 1909

L o eper: Sur quelques points de l'histologie normale et pathologique des plexus chorioides de l'homme. Comp. rend. de la Soc. Biol., T. 56, 1904.

Meek: A study of the choroid plexus. Journal of Comparative Neurol. and Psychol., Vol. XVII, Nr. 3, 1907.

Pettit et Girard: Processus secret. dans les cellules de revetement des plexus chor. des vent. lat.; consecutives a l'administ. de muscarin et d'ether. Comp. rend. de la Soc. Biol., T. 53, 1901.

Policard: Notes histophysiologiques sur la cellule hepatique. Comp. rend. de la Soc. Biol., 1909.

1) Diese Arbeiten waren mir nar in Auszügen bekannt. 
Prenant: Les mitochondries et l'ergastoplasme. Journ. de l'Anatom. et de la Phys., Nr. 3, 1910.

Rega ud et Na was: Sur les mitochondries des glandes salivaires chez les mammifères. Comp. rend. de la Soc. Biol., 1909.

Dieselben: Ergastoplasme et mitochondries dans les cellules de la glande sous-maxillaire de l'homme. Comp. rend. de la Soc. Biol., 1909.

Regaud: Participation du chondriome à la formation des grains de ségrégation dans les cellules des tubes contournes du rein. Comp. rend. de la Soc. Biol., 1909.

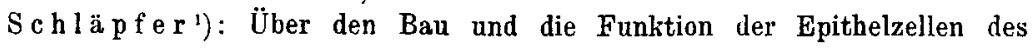
Plexus chorioideus Zi eglers Beiträge, VII, 1905.

$\mathrm{Studnička:} \mathrm{Untersuchungen} \mathrm{über} \mathrm{den} \mathrm{Bau} \mathrm{des} \mathrm{Ependyms} \mathrm{der} \mathrm{nervösen}$ Zentralnrgane. Anat. Hefte, H. XLVIII, 1900.

\section{Erklärung der Abbildungen auf Tafel X.}

Fig. 1. Katze. Schnitt durch den Plexus chorioideus des vierten Hirnventrikels. Behandlung nach Altmann. Reichert, homog. Immers. ${ }^{1 / 12}$. Okul. L e itz I. Eingeschobener Tubus. Abbildung vergrössert. $\mathrm{a}=$ Vakuolen; $\mathrm{b}==$ zweikernige Zelle; $\mathrm{c}=$ grosses fettähnliches Gebilde; $d=z$ wei Arten von Granula: 1 . fuchsinophile, 2. mit Osmiumsäure gefärbte; $e=$ Durchschnitt einer drüsenförmigen Einsenkung des Plexus chorioideus. Sämtliche Zellen enthalten fuchsinophile Zellen in verschiedener Menge.

Fig. 2. Katze. Das den Seitenventrikel bedeckende Epithel. Fixiert in dem modifizierten Gemisch von Al t $\mathrm{ma} n \mathrm{n}$. Färbung mit Hämatoxylin nach $\mathrm{H}$ e i d e $\mathrm{nh}$ a i n. $\mathrm{Z}$ e is s, Apochromat $1,30,2 \mathrm{~mm}$. Comp. Okul. 8. Tubuslänge 160. Nitochondrien sowie eine geringe Menge von Granula sichtbar

Fig. 3. Kałze. Seitenventrikei. Fixierung, Färbung und Veryrösserung wie in Fig. 2. In der mittleren Zelle sind Mitochondrien und kleine Granula, in den seitlich gelegenen eine grössere Anzahl von Granula sichtbar.

Fig. 4. Maus. Seitenventrikel. Fixierung und Färbung nach $\mathrm{A} 1 \mathrm{tm}$ a $\mathrm{n} n$. Apochromat $\mathrm{Z}$ e is s $1,30,2 \mathrm{~mm}$. Okul. $\mathrm{R}$ e ichert 4 . Tubuslänge 160. Fuchsinophile Körner.

Fig. 5. Pferd. Seitenventrikel. Fixierung und Färbung wie in Fig. 4. $\mathrm{Z}$ e is s, Apochromat 1,30, $2 \mathrm{~mm}$. Comp.-Oknl. 8. Tubusiänge 160 . Fuchsinophile Körner; Halbmondkörperchen; Vakuolen; fettähnliche Gebilde.

Fig. 6. Katze. Vierter Ventrikel. Fixierung und Färbung wie in Fig. 2. Apochromat $\mathrm{Z}$ e is s $1,30,2 \mathrm{~mm}$. Comp.-Okul. 8.

Fig. 7. Katze. Seitenventrikel. Behandlung wie in Fig. 2. $\mathrm{Z}$ e is s, Apochromat 1,30, $2 \mathrm{~mm}$. Comp.-Okul. 8. Tubuslänge 160 .

1) Diese Arbeiten waren mir nur in Auszügen bekannt. 
244 W. Hworostushin: Zur Frage über den Bau etc.

Fig. 8. Affe. Seitenventrikel. Fixierung and Färbung nach Alt mann. $\mathrm{Z}$ e is s, Apochromat 1,30, $2 \mathrm{~mm}$. Comp.-Olul. 12. Tubuslänge 160 Zwei Arten von Granula.

Fig. 9. Affe. Vierter Ventrikel. Behandlung nach Alt mann; Tuschzeichnung. $\mathrm{Z}$ e is s, Apochromat 1,30, $2 \mathrm{~mm}$. Comp.-Okul. 12. Tubuslänge 160.

Fig. 10. Dasselbe Präparat. Vergrösserung wie in Fig. 7.

Fig. 11. Hase. Seitenventrikel. Oberflächenansicht. Färbung in Methylenblau: fixiert in molybdänsaurem Ammonium. Vergrösserung wie in Fig. 7. $\mathrm{a}=$ in Alkohol, aufgelöste fettähnliche Gebilde.

Fig. 12. Katze. Seitenventrikel. Frisches Gewebe in physiologischer Kochsalzlösung. Sofort nach Eröffnung des Seitenventrikels abgezeichnet. R e i c h e r t, homog. Immers. ${ }^{1}{ }_{12}$; Okul. $4 . \quad a=$ fettähnliche Gebilde. Überall sind verschieden grosse glänzende Körner sichtbar.

Fig. 13. Pferd. Seitenventrikel. Nervengeflecht im Plexus chorioideus. Gefärbt mit Methylenblau; fixiert in molybdänsaurem Ammonium. R e i c h e r t, homog. Immers. ${ }^{1 / 12}$; Comp.-Okul. $8 . \quad \mathrm{a}=$ Blutgefïsse; $\mathrm{b}=$ markhaltige Nervenfasern; $\mathrm{c}=$ vasomotorische Nervenfasern $d=$ marklose Nervenfasern. In der Tiefe ist das zarte subepitheliale Geflecht sichtbar. 


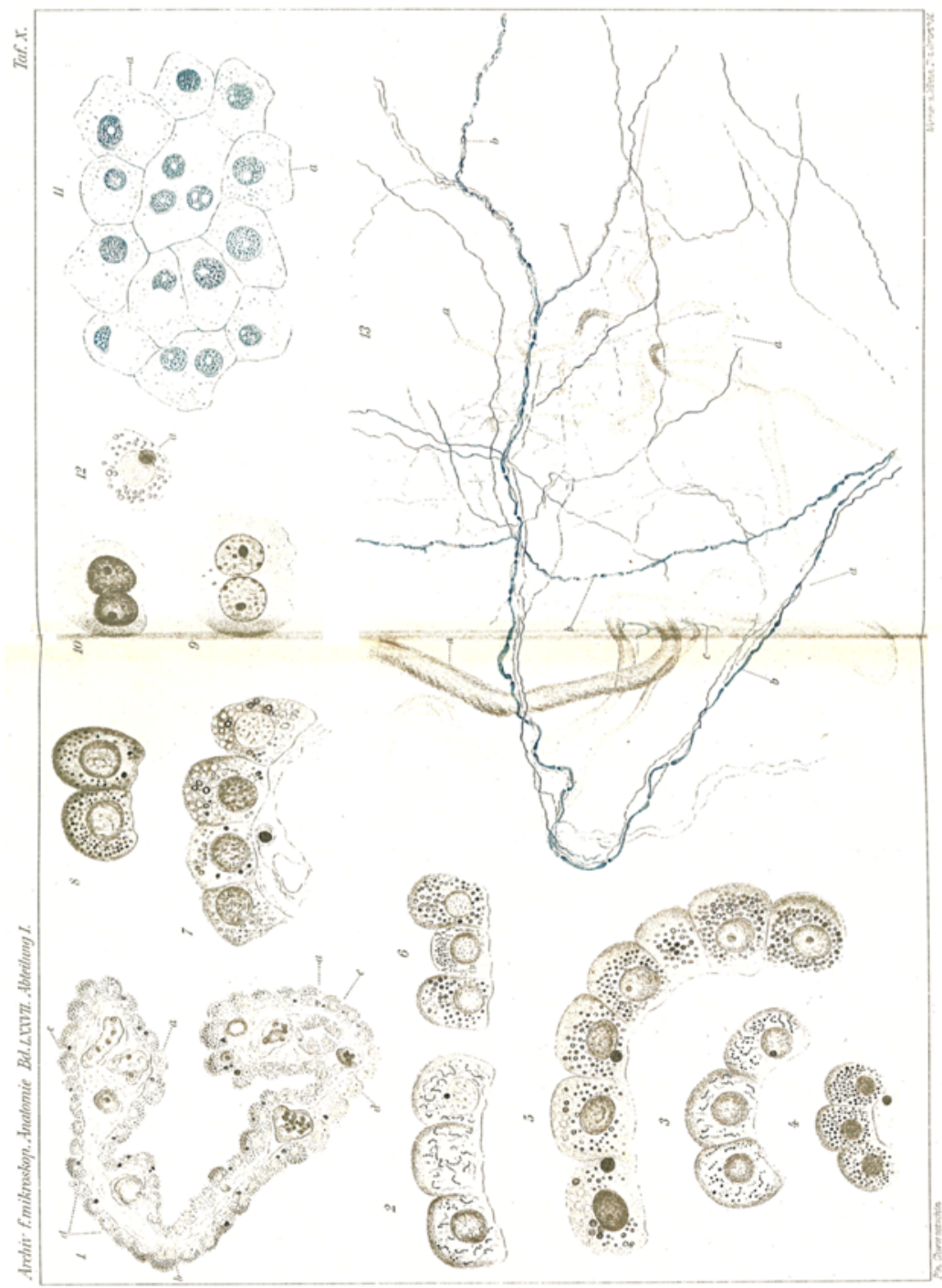

